\title{
Systematic Literature Review and Quantitative Analysis of Health Problems Associated with Sexually Transmitted Neisseria gonorrhoeae Infection
}

\author{
Jane Whelan · Jennifer Eeuwijk $\cdot$ Eveline Bunge $\cdot$ Ekkehard Beck
}

Received: March 15, 2021 / Accepted: June 10, 2021 / Published online: July 17, 2021

(C) The Author(s) 2021

\begin{abstract}
Objective: Neisseria gonorrhoeae $(\mathrm{Ng})$ is the second most common sexually transmitted bacterial infection (STI), leading to serious health problems in men, women and newborns. While early antibiotic treatment is effective, infections are increasingly antibiotic-resistant. No systematic reviews present health problems associated with $\mathrm{Ng}$ infections or their likelihood of occurrence. The objective, therefore, was to conduct a systematic literature review to address these gaps.

Methods: A systematic literature review was conducted of all studies with an English abstract published since 1950 (Pubmed)/1966 (Embase). The search included patients with a history of/ current sexually transmitted $\mathrm{Ng}$ infection. Expected outcomes were defined from published reviews of gonorrhoea health problems.
\end{abstract}

Supplementary Information The online version contains supplementary material available at https:// doi.org/10.1007/s40121-021-00481-z.

J. Whelan $(\square)$

GSK, Amsterdam, The Netherlands

e-mail: whelanjane@hotmail.com

J. Eeuwijk · E. Bunge

Pallas Health Research and Consultancy, Rotterdam, The Netherlands

E. Beck

GSK, Wavre, Belgium
Observational studies with a control group were included. A decision tree determined the best quality studies for each outcome, prioritising generalisable populations, laboratory-confirmed diagnosis, clearly defined outcomes, no STI co-infections, adjusted analyses and risk estimates. Where feasible, a meta-analysis was performed; otherwise, the best quality study estimates were identified.

Findings: In total, 46 studies were included, and 22 health problems were identified. Of these problems, $\mathrm{Ng}$ infection was statistically significantly associated with preterm premature ruptures of membranes, preterm birth, low birth weight, stillbirth, infant death, neonatal ophthalmia, schizophrenia in offspring, pelvic inflammatory disease and subsequent tubal infertility, human immunodeficiency virus and prostate cancer/problems. High-quality evidence was generally lacking, with high heterogeneity across studies, and limited or inconclusive data on other health problems.

Conclusion: $\mathrm{Ng}$ infection is associated with severe health problems in women, men and newborns. More high-quality comparative studies are needed to address the limitations in current knowledge.

Keywords: Epidemiology; Gonorrhea; Neisseria gonorrhoeae; Prevalence; Sexually-transmitted disease 


\section{Key Summary Points}

Neisseria gonorrhoeae $(\mathrm{Ng})$ is a sexually transmitted disease that can lead to serious health problems in men, women and newborns.

It is treatable with antibiotics but antibiotic resistance is growing. Untreatable $\mathrm{Ng}$ could result in the reemergence of health problems not seen since the pre-antibiotic era.

We conducted a systematic literature review and meta-analysis to identify all health problems associated with gonorrhoea infection and to quantify their frequency.

In total, 46 studies were included, and 22 health problems were identified. More high-quality comparative studies are needed to address the limitations in current knowledge.

\section{DIGITAL FEATURES}

This article is published with digital features, including a graphical plain language summary, to facilitate understanding of the article. To view digital features for this article go to https:// doi.org/10.6084/m9.figshare.14754018.

\section{INTRODUCTION}

Neisseria gonorrhoeae $(\mathrm{Ng})$ is the second most commonly reported bacterial sexually transmitted infection (STI) and results in substantial morbidity and economic cost worldwide [1].

In both men and women, a large proportion of the $\mathrm{Ng}$ infections are asymptomatic [2-4]. Ng infections can, however, lead to a broad range of health problems. These can be serious, such as pelvic inflammatory disease, ectopic pregnancy, infertility and chronic pelvic pain for women; epididymitis and infertility for men; as well as arthritis, disseminated gonococcal infections and increased HIV susceptibility and transmissibility in both men and women [5-8]. There are also consequences for newborns of women with an $\mathrm{Ng}$ infection, who can develop neonatal conjunctivitis, blindness and, rarely, disseminated gonococcal infection $[5,8]$.

Primary gonorrhoea infection is frequently asymptomatic and undiagnosed and repeat infection is common [9]. Particularly in women, the first clinical presentation may be a more serious health problem such as pelvic inflammatory disease [10]. The severity of $\mathrm{Ng}$ infections and related health problems can be effectively reduced or prevented when infected people receive early antibiotic treatment. There is, however, an increase in gonorrhoea infections that are resistant to currently available antibiotics [11]. If $\mathrm{Ng}$ infections become untreatable, the burden of HIV and other serious health outcomes is also expected to increase substantially, along with their associated healthcare costs [12]. Prevention of Ng infections can therefore play an important role in protecting public health and reducing the disease burden and healthcare costs.

There are no systematic reviews to date of published studies reporting on the occurrence of health problems associated with $\mathrm{Ng}$ infections or quantifying the frequency of occurrence of these health problems. Evidence on the burden of gonorrhoea infection in population subgroups at risk will be critical for the successful development and implementation of preventive measures $[13,14]$.

The objective was to conduct a systematic literature review to answer the following research questions: (1) What are the health problems associated with a primary $\mathrm{Ng}$ infection in the general population and in subgroups (i.e., heterosexual women and men, men who have sex with men, pregnant women and newborns), and what are the risks of developing these problems? Additional research questions were: (2) What are the relationships between different health problems associated with sexually transmitted $\mathrm{Ng}$, i.e., is there a common pathogenesis [e.g., the relationship between pelvic inflammatory disease (PID) and chronic pelvic pain, ectopic pregnancy and infertility in 
women, or infertility following epididymitis in men]? and (3) What are the potential gaps in the published evidence on health problems associated with an $\mathrm{Ng}$ infection? (Fig. 1).

\section{METHODS}

A systematic literature review was conducted following the Cochrane methodological guidelines [15] and Preferred Reporting Items for Systematic Review and Meta-Analyses (PRISMA) reporting guidelines [16]. Studies of interest to address the research questions were identified using the PICOS method (i.e., defined according to Population, Intervention, Comparison, Outcome, Study design).
The population of interest was defined as patients of all ages with a history of or with a current sexually transmitted $\mathrm{Ng}$ infection. As the literature on $\mathrm{Ng}$ health problems was expected to be limited, studies on health problems in Chlamydia trachomatis (Ct) patients, where $\mathrm{Ng}$ infections were also reported, were included-Ct-only populations were excluded. There were no Interventions or Comparisons of interest specified. The outcomes of interest were health problems found in both men and women (e.g., infertility, sepsis, meningitis, conjunctivitis, endocarditis, peritonitis, perihepatitis, polyarthralgia, arthritis, urethral stenosis, urethral stricture, proctitis, proctocolitis, HIV), health problems found in women alone (e.g., PID or salpingitis, ectopic

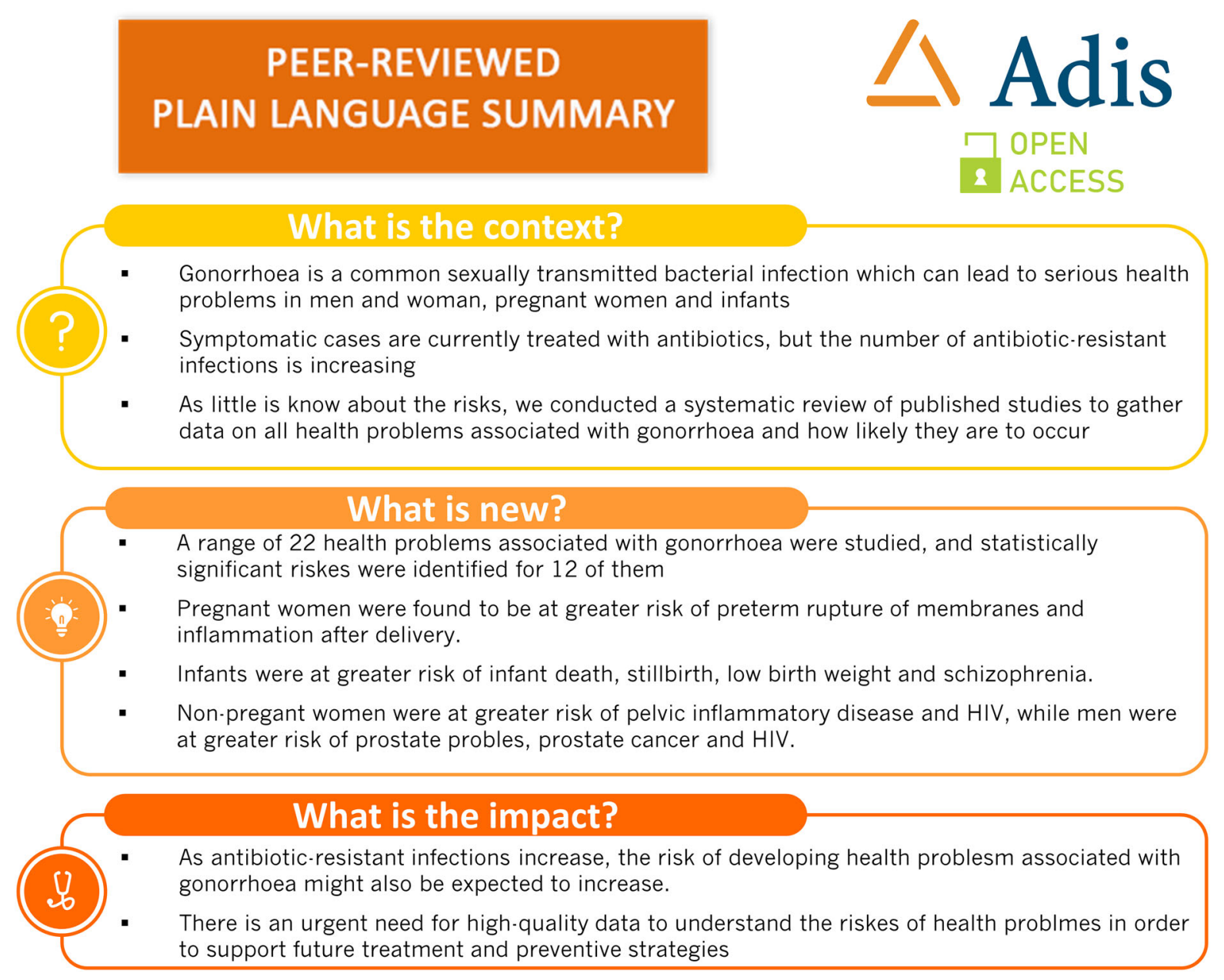

Fig. 1 Plain language summary 
pregnancy, bartholinitis, endometritis, tuboovarian abscess), in men alone (e.g., prostatitis and epididymitis) or in newborns (e.g., ophthalmia neonatorum), and any other health problem or outcome associated with $\mathrm{Ng}$ or $\mathrm{Ct}$ infection. The list of possible outcomes was based on extensive overviews of gonorrhoea health problems published by the Centres for Disease Control and Prevention (CDC) [5, 17] and the Dutch National Coordination Centre for Communicable Disease Control $[6,18]$. The study designs of interest were observational studies that included a comparator group. Case reports, case series, editorials and reviews were excluded. Systematic reviews and meta-analyses were included to search their reference lists for relevant primary studies that may have been missed by the search.

\section{Search and Selection Procedures}

Medline (accessed through PubMed) and Embase databases were searched by combining disease terms for $\mathrm{Ng}$ and $\mathrm{Ct}$ with a broad list of outcome terms for the possible health problems. Human studies, studies in any language with an abstract in English, primary studies and reviews published since 1950 (Pubmed) or 1966 (Embase) were included (see Appendix 1 Search strategies). Searches were conducted on July 24 , 2019.

All titles and abstracts were screened independently by two reviewers using pre-defined inclusion and exclusion criteria (Appendix 1 Table 1). The full text of selected papers was screened (first $10 \%$ independently by two researchers). Studies were excluded if they did not answer one of the research questions or were narrative reviews, had a small sample size (under 25 cases) or did not present results separately for $\mathrm{Ng}$ and $\mathrm{Ct}$. Furthermore, infection with $\mathrm{Ng}$ and/or Ct must already have been established; therefore, studies with outcome and infection determined at the same point in time were excluded. The reason for exclusion was recorded at each stage of the selection process.

The Scottish Intercollegiate Guidelines Network (SIGN) methodology checklists for observational studies were used to assess the quality of each included study. High-quality studies rated $(++)$ had little or no risk of bias, "acceptable-quality" studies rated as $(+)$ had some flaws with an associated risk of bias, while low-quality studies rated as (-) had significant flaws relating to key aspects of the study design.

Detailed data from each included study were entered into a predefined data extraction table in Excel (Appendix 1 Data extraction) along with the major limitations of the study and the basis for the SIGN score. Data were extracted by one researcher and checked by another. This article is based on previously conducted studies and does not contain any new studies with human participants or animals performed by any of the authors.

\section{Data Analysis}

The feasibility of conducting a meta-analysis was assessed. A preliminary review of the data showed that it was not possible to pool data from all studies per outcome because of high heterogeneity observed across studies. For example, some studies provided crude estimates while others adjusted for confounders, and there were differences in terms of method of diagnosis (e.g., self-report versus laboratoryconfirmed) or definition and reporting of outcome measures [e.g., hazard ratio (HR) versus incidence rate-ratio (IRR)].

For each outcome, therefore, a 'best estimate' was determined. This could either be a single best estimate from the highest quality study, in case data from studies reporting on the same outcome could not be pooled, or a pooled estimate of two or more studies considered to be of equal high-quality, i.e., not all studies identified were used to derive a pooled estimate. To decide whether studies could be pooled, and which studies provided the best evidence, a decision tree (Fig. 2) was derived iteratively as data extraction progressed. In brief, generalisable studies conducted in the general population with laboratory-confirmed gonorrhoea, clearly defined outcomes and analyses adjusted for confounding provided better quality evidence than studies in specific high-incidence 


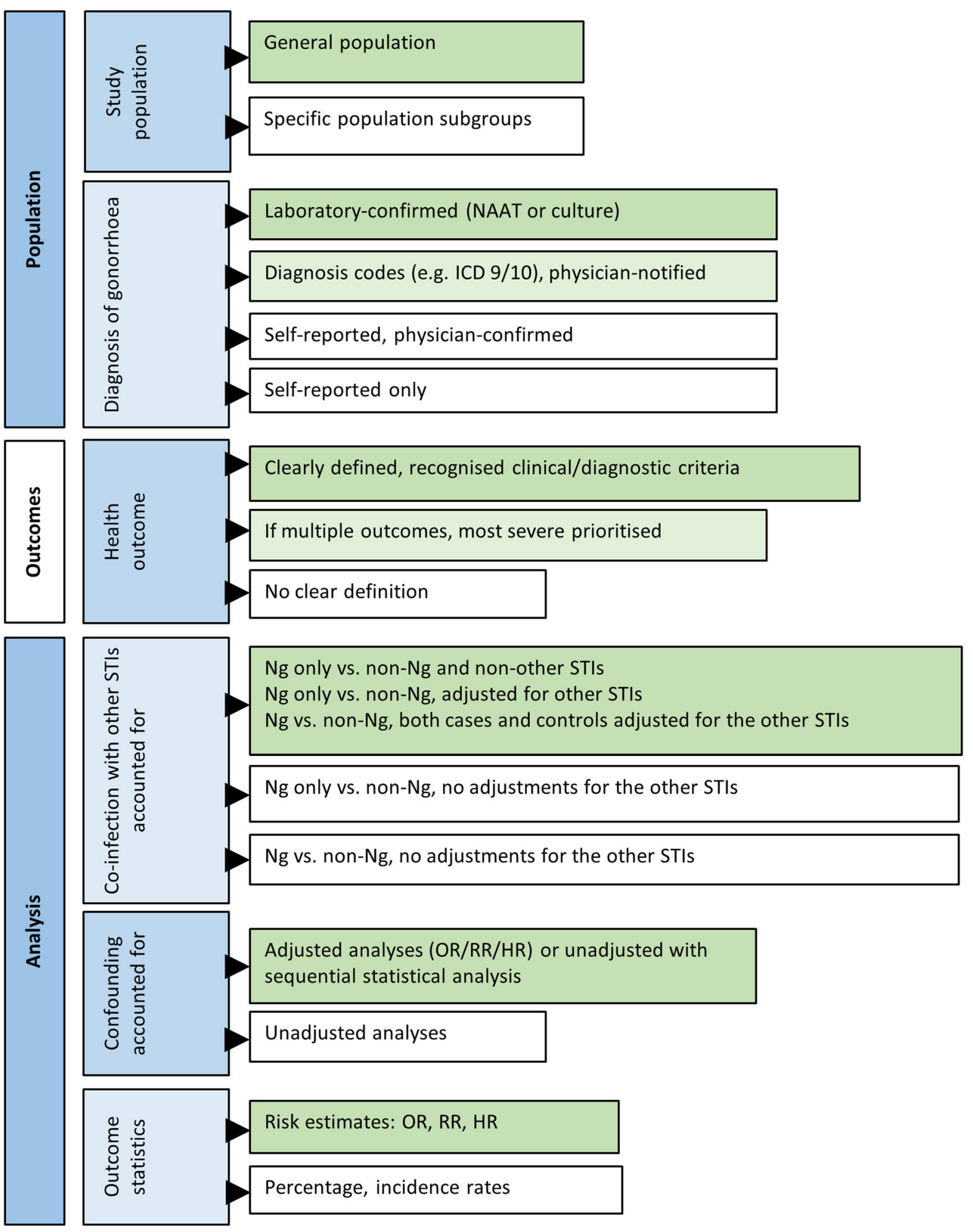


4Fig. 2 To illustrate the application of the decision tree: for each health outcome, studies that were of equivalent high quality on all elements of the decision tree were pooled. Any study that was not comparable on all elements was reported separately. HR, hazard ratio; OR, odds ratio; NAAT, nucleic acid amplification test; $\mathrm{Ng}$, Neisseria gonorrhoea; RR, risk ratio; STI, sexually transmitted infection

population subgroups, self-reported gonorrhoea or studies without adjustment for important confounders. Studies that compared populations with only gonorrhoea to populations without STIs were preferred to studies including populations with gonorrhoea and other STIs. If crude rates were presented but a rationale showed that adjustment was not necessary (e.g., because univariate analysis showed no significant results in a stepwise analysis), then adjusted and unadjusted odds ratio (OR)/risk ratio (RR)/HR were combined for that specific health outcome. If only crude numbers were provided and considered equal, they were pooled to provide a crude OR or RR with confidence intervals (CI). Where outcomes were not comparable, the most severe outcome was selected. Due to the limited number of studies identified, studies that were not considered for the best estimate or for meta-analysis were still evaluated for their consistency with the best estimate when judging the overall effect.

If included studies were comparable in terms of study population, exposure and outcome, then meta-analytic techniques were used to calculate pooled estimates, taking heterogeneity between studies into account with a randomeffects model. Heterogeneity was assessed by using the $I^{2}$ test and visual assessment of the forest plots. When available, adjusted ORs or RRs (aOR or aRR) were used to calculate the pooled OR or RR.

\section{RESULTS}

In total, 46 studies were included in this systematic review from 16,081 unique titles reviewed (Fig. 3). There were 19 studies in pregnant women with or without outcomes in newborns (all general-population samples), 16 studies in women (5 in high-incidence subgroups), 11 in men (7 in high-incidence subgroups) and 1 study in both men and women (general population sample).

The majority were cohort studies $(n=33)$, designed as retrospective or prospective cohort studies, surveillance studies or populationbased linkage of registry data. There were 12 case-control studies, including two nested casecontrol studies. One case series was included.

More than half of the studies were performed in the US $(n=28)$. The other studies were performed in Australia $(n=6)$, Kenya $(n=4)$, Canada $(n=3)$, UK $(n=1)$, Denmark $(n=1)$, Taiwan $(n=1)$, South Africa $(n=1)$, Ethiopia $(n=1)$ and Congo $(n=1)$.

Figure 4 shows that most of the evidence was in pregnant women with a range of outcomes but primarily focussing on preterm birth and stillbirth. Ten studies focussed on the risk of HIV in both women and men [including highincidence subgroups such as female sex workers and men who have sex with men (MSM)]. In women, PID risk was assessed in five studies. Overall, 22 studies were published in 2010-2019, 7 in 2000-2009, 11 in 1990-1999, 5 in 1980-1989 and 1 in 1976. Most of the outcomes in pregnant women and women were also studied in the last 10 years whereas recent evidence was only available for HIV and prostate cancer in men.

On application of the decision tree, the number of studies available for pooling was limited. All the studies selected for pooling were assessed to be of acceptable or low quality.

\section{Findings in Pregnant Women and Newborns}

All of the 19 studies in which gonorrhoea was diagnosed in pregnant women were conducted in general population samples and a broad range of 13 outcomes was assessed, some from a single study (Fig. 4).

For premature rupture of membranes (PROM), four studies were identified but could not be pooled as they presented different outcomes, i.e., preterm PROM (significant 
association found), prolonged PROM (non-significant association), full-term PROM (non-significant association), premature ruptures and 24-h ruptured membranes (both significant associations). Three studies [19-21] found an increased risk of PROM after gonorrhoea infection while one [22] did not. Ekwo et al. [19] and Heumann et al. [20] reported on population- based samples with laboratory-confirmed diagnosis, a clearly defined clinical endpoint and a statistical analytic approach that accounted for confounding. Of the two, Ekwo was selected as the best estimate as preterm PROM was reported, which is associated with high perinatal morbidity and mortality along with maternal

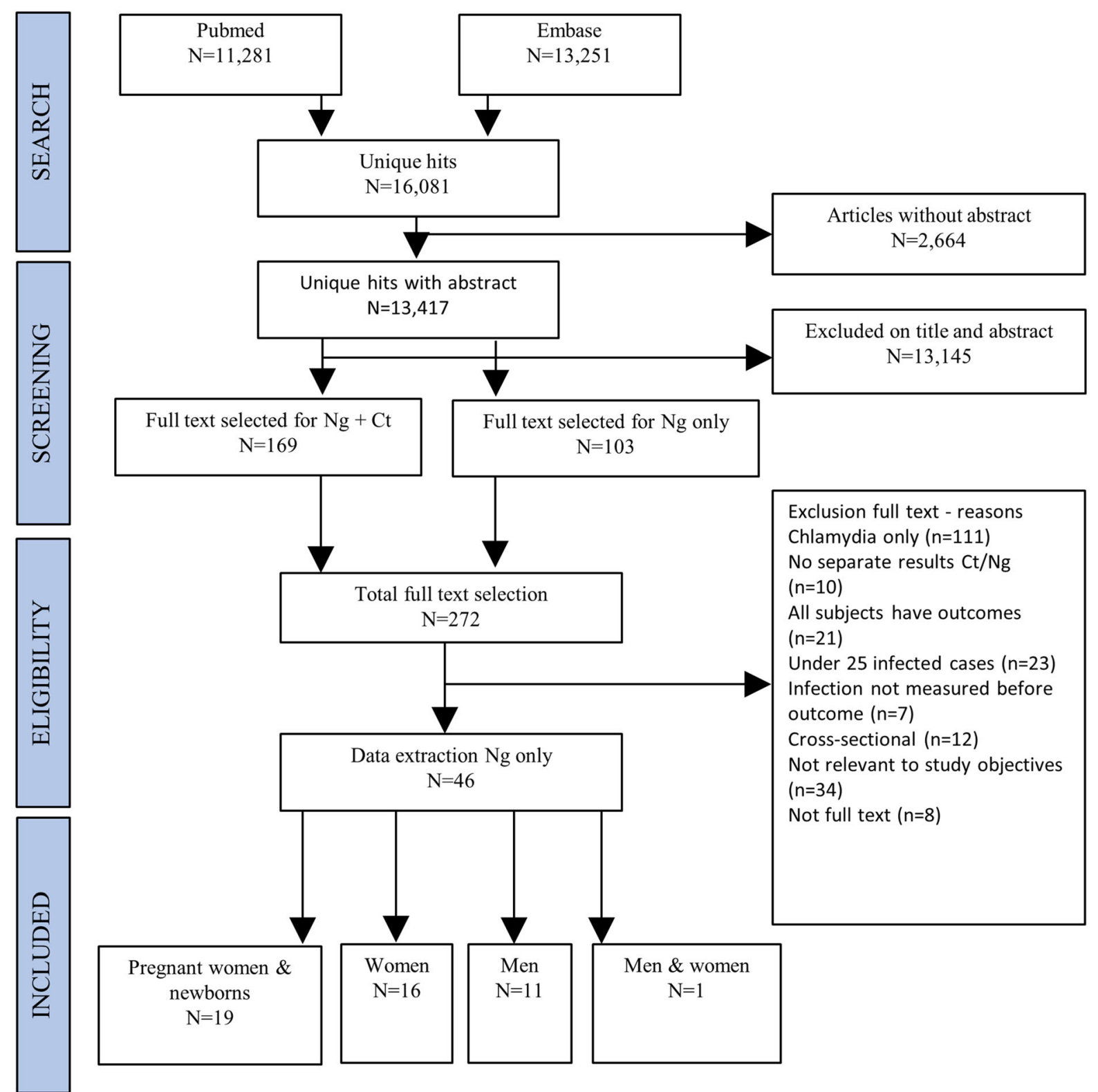

Fig. 3 PRISMA flow diagram 


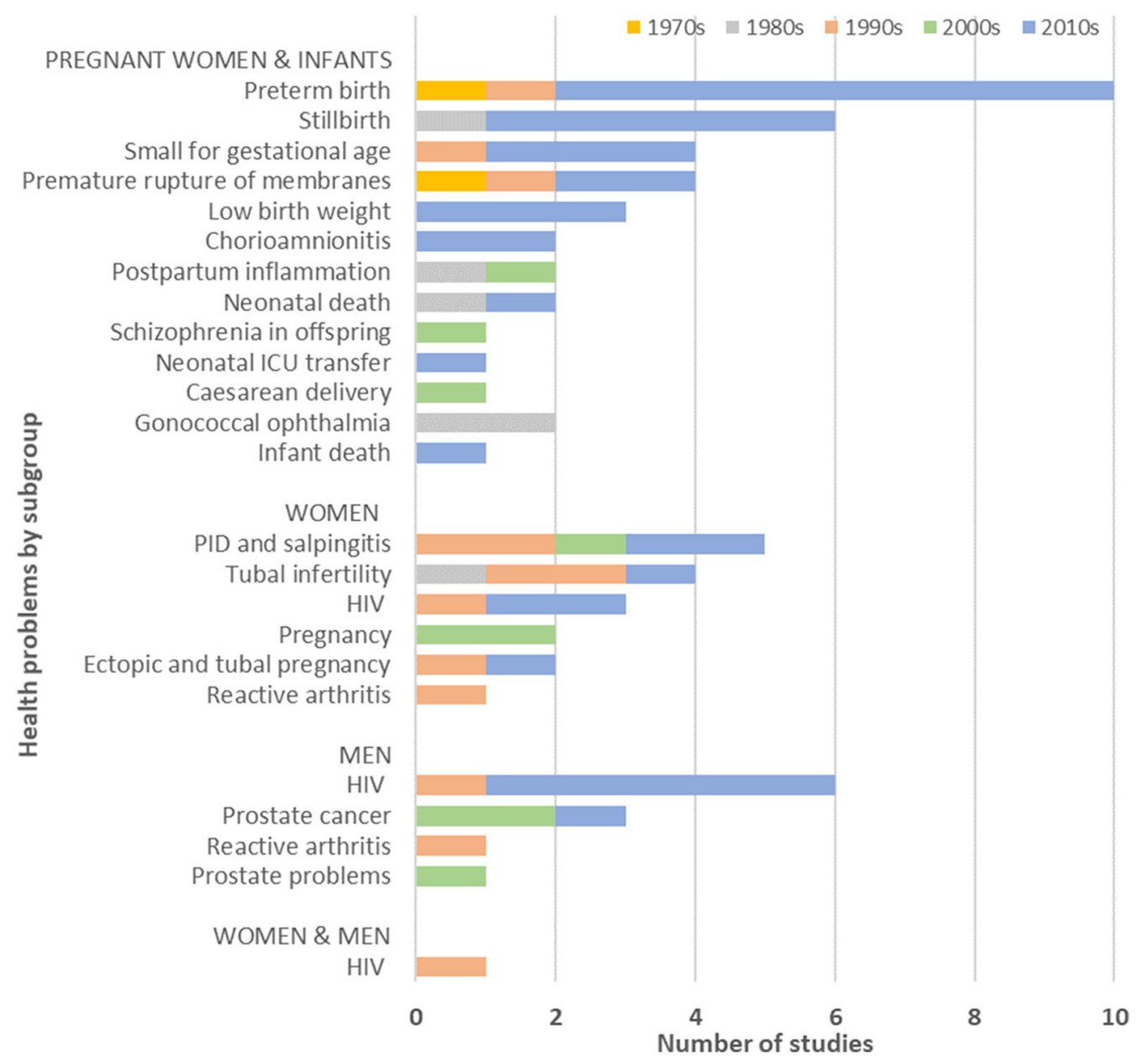

Fig. 4 Frequency of health problems studied in the literature by subgroup over time

morbidity, while Heumann studied the risk of prolonged PROM.

For preterm birth, five of the ten identified studies were considered sufficiently comparable for data to be pooled, according to the decision tree criteria. Eight studies [19-21, 23-27] found an association between gonorrhoea and preterm birth, four were statistically significant associations, while one study [22] found no association (non-significant) and another study [28] reported preterm births in pregnant women with disseminated gonococcal infection but had no control group.

For stillbirth, there were six studies, of which two were pooled. There was too much heterogeneity among the remaining studies in study outcomes and populations and lack of adjustment for coinfection and other factors. Three studies [23, 24, 29] found an association between maternal gonorrhoea infection and stillbirth, one a significant association [24] and two $[27,30]$ presenting crude percentages found no association (non-significant).

A non-statistically significant increased risk of neonatal death following maternal gonorrhoea infection was observed in two studies in a general population sample with laboratoryconfirmed diagnosis using different outcome statistics [30, 31]. Schonfeld et al. [31] were found to provide the best estimate of risk (i.e., use of OR instead of crude percentages), reporting a wide $\mathrm{CI}$ and positive point effect far from the null.

A significant association was found between maternal gonorrhoea infection and infant death in a single, large and good-quality study [29]. 
An increased risk for "small for gestational age" (SGA) after maternal gonorrhoea infection was observed in four studies [20, 23, 27, 32] using different outcome statistics. The association was non-statistically significant in two studies [23, 32] and significance was not

Table 1 Pooled and single best estimates of the risk of health problems associated with gonorrhoea in pregnant women and newborns

\begin{tabular}{|c|c|c|}
\hline $\begin{array}{l}\text { Outcome (*statistically } \mathrm{R} \\
\text { significant) }\end{array}$ & Result & Pooled/single best \\
\hline \multicolumn{3}{|c|}{ Pregnant women and newborns } \\
\hline${ }^{*}$ Preterm PROM & $\begin{array}{l}\text { aOR: } 7.6(95 \% \mathrm{CI} \\
2.2-26.4)\end{array}$ & Single best: Ekwo et al. [19] \\
\hline${ }^{*}$ Infant death & $\begin{array}{l}\text { aHR: } 3.83(95 \% \text { CI } \\
1.16-12.68)\end{array}$ & Single best: Warr et al. [29] \\
\hline${ }^{*}$ Schizophrenia in offspring & $\begin{array}{c}\mathrm{g} \text { aOR: } 3.41(95 \% \mathrm{CI} \\
1.36-8.57)\end{array}$ & Single best: Sorensen et al. [37] \\
\hline *Stillbirth & $\begin{array}{l}\text { aOR: } 2.24(95 \% \mathrm{CI} \\
1.12-4.46)\end{array}$ & Pooled: Liu et al. [23], Moodley et al. [24] \\
\hline $\begin{array}{l}{ }^{*} \text { Postpartum inflammation } \\
\quad(\text { PID after delivery })\end{array}$ & $\begin{array}{l}\text { aHR: } 2.0(95 \% \mathrm{CI} \\
1.3-3.1)\end{array}$ & Single best: Mahon et al. [35] \\
\hline${ }^{*}$ Preterm birth & $\begin{array}{l}\text { aOR: } 1.36(95 \% \mathrm{CI} \\
1.07-1.72)\end{array}$ & $\begin{array}{l}\text { Pooled: Liu et al. [23], Moodley et al. [24], Heumann et al. [20], } \\
\text { Waight et al. [25], Ekwo et al. [19] }\end{array}$ \\
\hline${ }^{*}$ Low birth weight & $\begin{array}{l}\text { aOR: } 1.17(95 \% \mathrm{CI} \\
\quad 1.02-1.33)\end{array}$ & Pooled: Waight et al. [25], Moodley et al. [24], Heumann et al. [20] \\
\hline Neonatal death & $\begin{array}{l}\text { cOR: } 2.48(95 \% \mathrm{CI} \\
0.68-8.98)\end{array}$ & Single best: Schonfeld et al. [31] \\
\hline Small for gestational age & $\begin{array}{l}\text { aOR: } 1.33(95 \% \mathrm{CI} \\
0.84-2.12)\end{array}$ & Pooled: Liu et al. [23], Heumann et al. [20] \\
\hline Neonatal ICU transfer & $\begin{array}{l}\text { aOR: } 1.2(95 \% \text { CI } \\
0.9-1.7)\end{array}$ & Single best: Heumann et al. [20] \\
\hline Chorioamnionitis & $\begin{array}{l}\text { cOR: } 0.8(95 \% \mathrm{CI} \\
0.6-1.2)\end{array}$ & Single best: Heumann et al. [20] \\
\hline Caesarean delivery & $\begin{array}{l}18.4 \% \text { vs. } 23.3 \% \\
P=0.4279\end{array}$ & Single best: Hill et al. [22] \\
\hline $\begin{array}{l}\text { Gonococcal ophthalmia } \\
\text { (no prophylaxis) }\end{array}$ & $42 \%$ & Single best: Laga et al. [33] \\
\hline $\begin{array}{l}\text { Gonococcal ophthalmia } \\
\text { (with prophylaxis) }\end{array}$ & $0 \%$ & Single best: Hammerschlag et al. [34] \\
\hline No literature found & NA & \\
\hline
\end{tabular}

aHR adjusted hazard ratio, $a O R$ adjusted odds ratio, PROM premature rupture of membranes, $c O R$ crude odds ratio 
reported in one [27]. Findings of Liu et al. [23] and Heumann et al. [20], both reporting ORs and accounting for coinfection, were pooled.

Maternal gonorrhoea infection was associated with an increased risk of low birth weight in two studies [20, 25] (one significantly associated [20]) and one non-significant association, though with a point estimate below the null (non-significant) in another study [24]. Studies were comparable enough in terms of population, outcome definition, adjusted analyses and outcome statistics to be pooled.

For chorioamnionitis [20, 22] and caesarean delivery [22], no relation was observed between maternal gonococcal infection and outcome. For the risk of chorioamnionitis, Heumann et al. [20] was selected over Hill et al. [22] as the estimate of risk (OR) was considered superior to percentages.

Gonococcal ophthalmia was strongly related with maternal infection in one study which found that ophthalmia neonatorum developed in 28 out of 67 babies whose mothers had gonorrhoeae infections, a transmission rate of $42 \%$ [33]. It was, however, very effectively prevented with prophylactic treatment in another study [34].

Two studies [35, 36] showed a significant association between gonorrhoea infection and various outcomes relating to inflammation after delivery. Plummer et al. [36] studied risk of salpingitis and endometritis within 1 month after delivery while Mahon et al. [35] studied PID within 1 year after delivery, excluding cases within 1 month of delivery. Mahon provided the best risk estimate, use of adjusted analyses and reliable outcome statistics.

A significant association was found between gonorrhoea during pregnancy and schizophrenia in the offspring in a large single study with self-reported diagnosis and adjusted analyses using OR [37].

Table 1 summarises the single best estimate or pooled estimate for each outcome. Significant associations were observed for preterm PROM, infant death, schizophrenia in offspring, stillbirth, postpartum inflammation, preterm birth, and low birth weight and gonococcal ophthalmia. Outcomes that were non-significant, but for which the data were compatible with a potential increased risk (point estimate $>1$ ) were neonatal death, SGA and neonatal intensive care unit (ICU) transfer. Of the predefined list of possible health outcomes in pregnant women and newborns, all were explored in at least one study (Table 1).

For all 19 studies in pregnant women and/or newborns, publication details, study design and outcomes are summarised in Appendix 3 Table 1, with an evaluation of the sources of heterogeneity and rationale supporting the use of a single best estimate (green) or pooled estimate (orange) for each outcome. The forest plots for pooled estimates are presented in Appendix 2.

\section{Findings in Women and Men}

In total, 16 studies were included on gonococcal infection in women, of which 6 were conducted in high-incidence subgroups (high chlamydial risk based on a risk score) [38], sex workers $[39,40]$, high risk of lower genital tract infection [41], Aboriginal and Torres Strait Islander women [42] and women attending an STI clinic [43]). Six outcomes were assessed (four in the last 10 years); PID and salpingitis, tubal or ectopic pregnancy, infertility or incidence of pregnancy, HIV seroconversion and reactive arthritis (Fig. 4).

Significant associations were observed for PID and HIV in women with gonorrhoea, while non-significant associations were observed for ectopic and tubal pregnancy, pregnancy and tubal infertility (except for women with a history of PID). No literature was identified for the following outcomes in women: bartholinitis, endometritis, tubo-ovarian abscess (Table 2).

Gonorrhoea infection in women was significantly associated with a higher risk for PID. Five studies were included on the development of PID or salpingitis [10, 38, 39, 44, 45], but studies could not be pooled as outcome statistics were not compatible and coinfection was not adjusted for. Reekie et al. [44] provided the best estimate from a large study with laboratorytested gonorrhoea, IRR calculated for women with only $\mathrm{Ng}$ infection and analysis adjusted for risk factors. 
Table 2 Pooled and single best estimates of the risk of health problems associated with gonorrhoea in women and men

\begin{tabular}{lll}
\hline $\begin{array}{l}\text { Outcome (*statistically } \\
\text { significant) }\end{array}$ & Result & Best/pooled
\end{tabular}

Women

*Pelvic inflammatory disease

${ }^{*} \mathrm{HIV}$

Ectopic and tubal pregnancy

Pregnancy (STI clinic attendees)

Tubal infertility

Reactive arthritis

No literature found

Men

${ }^{*}$ Prostate cancer

*Prostate problems

Total prevalent LUTS

Total incident LUTS

Surgery for enlarged prostate

${ }^{*} \mathrm{HIV}$

MSM with only rectal gonorrhoea

MSM with only rectal gonorrhoea, no comparator

Reactive arthritis

No literature found

Mixed population (men and women)

${ }^{*} \mathrm{HIV}$

No literature found
aIRR: 4.54 (95\% CI 3.87-5.33)

cRR: 2.57 (95\% CI 1.42-4.64)

aRR: 1.15 (95\% CI 0.82-1.62)

aHR: 1.3 (95\% CI 0.8-2.0)

aIRR: 1.38 (95\% CI 0.67-2.86)

$3.8 \%$ vs. $0 \%$

Bartholinitis, endometritis, tubo-ovaan abscess

aHR: 5.66 (95\% CI 1.36-23.52)

Best: Wang et al. [53]

Best: Sutcliffe et al. [56]

aOR: 1.76 (95\% CI 1.43-2.15)

aOR: 1.63 (95\% CI 1.14-2.33)

aOR: 1.49 (95\% CI 1.06-2.08)

aOR: 2.3 (95\% CI 1.4-3.9)

Best: Barbee et al. [57]

cIncidence: 7.10 (95\% CI

$3.30-13.49)$

$0 \%$ vs. $0 \%$

Prostatitis, epididymitis, infertility

aOR: 2.8 (95\% CI 2.12-3.69), $P<0.001$

Sepsis, meningitis, conjunctivitis, endocarditis, peritonitis, perihepatitis, polyarthralgia, arthritis, urethral stenosis, urethral stricture, proctitis, proctocolitis

aHR adjusted hazard ratio, $a I R R$ adjusted incidence rate-ratio, $a O R$ adjusted odds ratio, $c R R$ crude risk ratio, $H I V$ human immunodeficiency virus, $S T I$ sexually transmitted infection, $a R R$ adjusted risk ratio

Non-significant associations were observed for gonorrhoea and ectopic or tubal pregnancy based on pooled data from two studies [46, 47], but the data were compatible with an increased risk (point estimate $>1$, but wide $\mathrm{CI}$ ). One large study in nucleic acid amplification test (NAAT)- 
diagnosed women comparing only Ng-positive, $\mathrm{Ng}$-positive and women with no STIs found an increased point estimate (non-significant) of ectopic pregnancy [46], while Sherman [47] found a lower risk of tubal pregnancy (nonsignificant) in a case-control study using selfreported physician-made diagnosis. Studies could be pooled as $>90 \%$ of all ectopic pregnancies were tubal ectopic pregnancies.

Two studies using laboratory-diagnosed gonorrhoea in high-incidence subgroups reported on pregnancy after $\mathrm{Ng}$ infection [41, 42]. As Aboriginal and Torres Strait Islander women are a minority population of Australia, Wiesenfeld's study [41] in women attending STI clinics in the US was considered more generalisable to other populations and was selected as the best estimate.

The association between gonorrhoea infection and tubal infertility was non-significant; however, the point estimate was $>1$ and the data were compatible with an increased risk. Four studies were included [46, 48-50]: Reekie et al. [46] provided the best estimate, using laboratory-confirmed gonorrhoea, while the other studies included self-reported diagnosis. The pooled estimate of these other studies was comparable to the best estimate. In the study by Cates et al. [50], the risk of tubal infertility was significantly higher [aOR $12.6 \quad(95 \%$ CI 5.6-28.1)] in women with overt (i.e., with a history of) PID (11/34 had Ng infection) than in the control group (31/1598 had Ng infection).

Gonorrhoea infection in women was associated with a significantly increased risk of HIV seroconversion. Three studies were included $[40,51,52]$, one in sex workers [40], and all three reported an increased risk. Data from the two large studies using a general-population sample were pooled.

Reactive arthritis was reported in one case with culture-diagnosed gonorrhoea in a small study $(N=76)$ following up women attending an STI clinic [43].

Individual study details for women can be found in Appendix 3 Table 2 with forest plots for pooled estimates in Appendix 2.

In total, 11 studies in men were included on gonorrhoea infection, covering outcomes of prostate problems (in a population of health professionals), prostate cancer (two studies in populations of health professionals and African American men), HIV seroconversion (all studies in populations of MSM) and reactive arthritis (in a population of men attending an STI clinic). Recent studies from the last 10 years assessed prostate cancer and HIV (Fig. 4).

Significant associations were observed for prostate cancer, prostate problems (lower urinary tract symptoms) and HIV seroconversion. No literature was identified on the following outcomes: prostatitis and epididymitis (Table 2).

Gonorrhoea infection was significantly associated with prostate cancer in a large general-population sample study [53] and a study in African American men [54], with a non-significant association found in health professionals [55]. The studies used different outcome statistics (HR, RR, OR) and adjusted for some different risk factors. Wang et al. [53] provided the best estimate as diagnosis was based on International Classification of Diseases-10 (ICD10) codes whereas the other studies used selfreported physician-made diagnoses.

Gonorrhoea infection was significantly associated with prostate problems, e.g., prevalent and incident lower urinary tract symptoms (LUTS) and surgery for an enlarged prostate, in a single large study in male health professionals [56].

Gonorrhoea infection was significantly associated with HIV seroconversion in MSM in all six studies included [57-62]. There were differences in study design, diagnosis methods (i.e., culture, NAAT and self-reported history) and study population infection site (e.g., two studies $[59,61]$ reported risks by rectal, pharyngeal or urethral $\mathrm{Ng}$ infection). Barbee et al. [57] provided the best estimate, despite only including MSM with rectal gonorrhoea; the analysis was adjusted for important risk factors and gonorrhoea diagnosis was laboratoryconfirmed.

No cases of reactive arthritis were reported in culture-diagnosed gonorrhoea or in controls with no STIs in a small study $(N=195)$ following up men attending an STI clinic [43].

Individual study details for men can be found in Appendix 3 Table 3. 
One study [63] was included that did not stratify results for men and women. Beck [63] found a significant association with HIV seroconversion in a large mixed population of both men and women with self-reported Ng infection (Table 2, Appendix 3 Table 4).

\section{DISCUSSION}

Until recently, gonorrhoea infection has been treatable with antibiotics, and in the industrialized world, research on complications related to gonorrhoea infection has been limited. Antibiotic resistance is growing, however, and gonorrhoea infection may become untreatable in the future. The World Health Organization (WHO) has called for a greater understanding of the gaps in our knowledge and data and research needs also with regards to disease outcomes, particularly as interest in the development of a gonococcal vaccine is renewed [64].

This systematic literature review on health problems associated with gonorrhoea infection identified 46 studies published between 1976 and 2019, of which 22 studies were published since 2010 . The majority of studies were in pregnant women and newborns, primarily focussing on preterm birth and stillbirth. HIV seroconversion in women and MSM was the next most frequently studied outcome.

A number of significant associations were identified by subgroup, based on pooled or single best estimates. In pregnant women, there was an increased risk of preterm PROM, infant death, schizophrenia in offspring, stillbirth, postpartum inflammation, preterm birth and low birth weight, and gonococcal ophthalmia without prophylaxis in general-population samples. In women with gonorrhoea, significant associations were observed for PID and HIV seroconversion (in both general and high-incidence subpopulations). In men with gonorrhoea, there were significant associations observed for prostate cancer (in both general and-high incidence subpopulations), prostate problems (in the general population) and HIV seroconversion (in the high-incidence MSM subpopulation). An updated meta-analysis published in 2015 of 21 observational studies also found a significant association between gonorrhoea infection and increased incidence of prostate cancer, particularly in African American men [65]. For PROM, preterm birth, stillbirth and prostate cancer, the evidence was less convincing as some studies showed a significant association, but in additional evidence this was not confirmed by all studies. For chorioamnionitis, caesarean delivery, neonatal ICU transfer, neonatal death, SGA, chance of pregnancy, ectopic or tubal pregnancy, none of the included studies showed a significant association with gonorrhoea infection. Data on reactive arthritis were not sufficient to draw conclusions.

To pool data, studies must be sufficiently comparable in terms of population, outcome and exposure assessed. Research methodologies and diagnostics have evolved since the 1970s resulting in heterogeneity across studies in terms of populations, outcomes and diagnostic measures, which have changed over such a large time frame, along with the use of different outcome statistics and adjustments for confounding. Therefore, it was not always possible to pool all data per outcome. When meta-analysis was not possible, the decision-tree approach was used to identify the best estimates from the highest-quality studies assessing each outcome (based on generalisability, diagnosis method, outcome definition and statistical methods). At times, the single best estimate identified for an outcome could differ from the findings of other studies assessing the same outcome, as these may have been from specific population subgroups or may have used self-reported rather than culture-defined diagnosis methods. In other cases, there was only one low-quality study assessing a particular outcome (e.g., reactive arthritis).

Several outcomes for which the pathogenesis was well understood and which were expected to be associated with gonorrhoea were either not identified in any of these controlled observational studies or findings were inconclusive; for women, these were endometritis, tuboovarian abscess or bartholinitis, while for men, no studies assessed prostatitis or epididymitis or infertility. In both men and women, no studies 
assessed the risk following $\mathrm{Ng}$ infection of sepsis, meningitis, conjunctivitis, endocarditis, peritonitis, perihepatitis, polyarthralgia, arthritis, urethral stenosis, urethral stricture, proctitis or proctocolitis. It is possible that these might be rare outcomes that were not picked up in these observational studies which excluded case reports and studies without a control group.

Regarding general population versus subgroup findings, most studies were in pregnant women. This may be because pregnant women are easier to recruit and follow up in studies than other population subgroups, such as MSM and sex workers. Only one study compared the risk in a high incidence population versus a general population directly; individuals who identified as MSM had a $4.94 \quad(95 \%$ CI 3.11-7.83) times higher HIV incidence rate compared to the non-MSM population after gonorrhoea diagnosis [62]. Because of the very limited amount of evidence, no conclusions could be drawn on increased risk of specific health problems in specific subpopulations, such as MSM.

Regarding the relationships between different health problems associated with Ng infection, only limited evidence was identified. Two studies analysed the association between $\mathrm{Ng}$ infection and tubal infertility, stratified by type of PID. One study stratified by history of PID [49]: the strongest association was found in women with a history of PID requiring hospitalisation, followed by a history of PID without hospitalisation and no association was found in women without a history of PID. Another study [50] stratified by women with 'atypical' PID (i.e., no past history of PID) and 'overt' (with a history of) PID; a strong association was found between gonorrhoea infection and tubal infertility in women with overt PID (aOR 12.6 [95\% CI 5.6-28.1]) but no association was found in women with atypical PID. Similarly, very limited information was available on the natural history and aspects of health problems associated with sexually transmitted gonorrhoea infections such as duration and time of onset relative to the primary gonorrhoea infection. We were not able to comment further on these aspects. Finally, for some expected outcomes for which causal pathogenic pathways have been clearly described in the literature, we were unable to find a statistically significant association or no evidence was identified.

Some key strengths of this systematic review are the use of a broad search strategy which resulted in a large variety of studies being identified in both the general population and high-incidence subpopulations. Rather than omit evidence that did not meet the criteria for meta-analysis, a systematic approach was taken to data summary, allowing a stepwise evaluation of each aspect of the study design: the study population, exposure, analysis and outcome. This approach resulted in a pooled or single best estimate for the relation of gonorrhoea infection with a specific health outcome, each set in the context of the other available evidence.

A number of limitations were noted. The limited number of studies for some health problems made it difficult to arrive at a coherent estimate for these outcomes. Included studies relating to each outcome varied in terms of design, population characteristics, health problem definitions, outcome statistics and diagnostic methods to determine gonorrhoea infection, which limited comparisons between the studies. As noted above, data on the interval between $\mathrm{Ng}$ infection and manifestation of health problems were limited. There was also a lack of high-quality evidence; studies were often not primarily designed to study the relation between gonorrhoea and health problems; there were limited adjustments in the analyses for health risk behaviour; no adjustments were possible in the analyses of rare health problems as the number of events was too low for adjusted analyses; and retrospective studies could miss asymptomatic cases. All the studies that were pooled for comparison were of low or acceptable quality, and the internal validity of studies selected for pooling may not be equivalent. Other limitations concern possible missed health problems, as a predefined list of problems was used in the search string, and because articles without abstracts were excluded, relevant (but likely older) articles could have been missed. Data from Europe (two studies) and Asia (one study) were limited. No studies were included from Latin America 
considering the inclusion criteria of English title and abstract.

\section{CONCLUSIONS}

Neisseria gonorrhoea is the second most commonly reported sexually transmitted bacterial infection and results in substantial morbidity and economic cost worldwide. Despite this, our review covering a period of 70 years identified only 13 studies that were of good quality, in which 14 different health problems associated with gonorrhoea infection were studied. No very-high-quality studies were identified.

Overall evidence on 22 health problems associated with $\mathrm{Ng}$ infection reported in 46 included studies was limited and highly heterogenous, further limiting the ability to pool study outcomes.

Statistically significant associations were identified for 12 health problems (i.e., preterm PROM, preterm birth, low birth weight, stillbirth, infant death, neonatal ophthalmia, schizophrenia in offspring, PID and tubal infertility after PID, HIV seroconversion, prostate cancer and prostate problems), but for others the evidence was inconsistent or insufficient. Some outcomes expected to be found in men, based on existing knowledge of causal pathogenic pathways, were not described in any of the included studies, e.g., infertility in men, prostatitis and epididymitis.

The WHO has called for improved global and regional estimates of $\mathrm{Ng}$-associated clinical and various other disease outcomes [64]; given the known pathogenesis of the infection, further high-quality prospective comparative studies conducted in the general population and in high-incidence subgroups are warranted to better understand the relationship between gonorrhoea infection and health problems, particularly for outcomes where a positive point estimate was accompanied by wide CI, and data were compatible with a wide range of effects from no effect to a potentially large effect. A large proportion of $\mathrm{Ng}$ infections are asymptomatic. Therefore, a screening study should be performed in a large group of people with and without symptoms. Information should be collected on other STIs and lifestyle factors (e.g., smoking) that could confound the association between gonorrhoea and health problems. Retrospective studies might be of sufficient quality, if they include a large number of subjects and laboratory-confirmed gonorrhoea diagnosis, if gonorrhoea infection is established independently from the outcome and if important risk factors are taken into account. These types of studies might be less complex and costly and could bring added value relatively quickly. The review of published studies has highlighted some important areas of improvement in future research. The decision tree, developed to evaluate the quality of studies for analysis, could be a useful reference when designing future studies, drawing attention to key elements of the study design that help to increase both internal and external validity.

As more comprehensive evidence on health outcomes associated with gonorrhoea becomes available, the search string of the present study should be updated to reflect this and the search re-run to ensure maximal representativeness in terms of health problems associated with $\mathrm{Ng}$ infection.

In the interim, while data on the natural history of infection are only partially available, novel analytic techniques (such as Bayesian multi-parameter evidence synthesis models) could be considered to arrive at more coherent or consistent estimates. Expert opinion to interpret the present findings and their applicability to health economic assessments of prevention interventions against $\mathrm{Ng}$ infections is also advised.

\section{ACKNOWLEDGEMENTS}

Funding. GlaxoSmithKline Biologicals SA was the funding source and was involved in all stages of the study conduct and analysis. GlaxoSmithKline Biologicals SA also funded all costs associated with the development and the publishing of the present manuscript.

Medical Writing and Editorial Assistance. The authors thank Dominique Rosillon 
(GSK) for discussion. The authors thank Business \& Decision Life Sciences platform for editorial assistance and manuscript coordination, on behalf of GSK. Kavi Littlewood (Littlewood Writing Solutions) provided medical writing support; Pierre-Paul Prévot coordinated manuscript development and editorial support.

Authorship. All named authors meet the International Committee of Medical Journal Editors (ICMJE) criteria for authorship for this article, take responsibility for the integrity of the work as a whole, and have given their approval for this version to be published.

Author Contributions. Jane Whelan, Jennifer Eeuwijk, Eveline Bunge and Ekkehard Beck participated to the concept, design, analysis, draft and review of this manuscript.

Disclosures. Ekkehard Beck and Jane Whelan are employees of the GSK group of companies and hold shares in it. Jennifer Eeuwijk and Eveline Bunge report grants from GSK Biologicals, during the conduct of the study and outside the submitted work.

Compliance with Ethics Guidelines. This article is based on previously conducted studies and does not contain any new studies with human participants or animals performed by any of the authors.

Open Access. This article is licensed under a Creative Commons Attribution-NonCommercial 4.0 International License, which permits any non-commercial use, sharing, adaptation, distribution and reproduction in any medium or format, as long as you give appropriate credit to the original author(s) and the source, provide a link to the Creative Commons licence, and indicate if changes were made. The images or other third party material in this article are included in the article's Creative Commons licence, unless indicated otherwise in a credit line to the material. If material is not included in the article's Creative Commons licence and your intended use is not permitted by statutory regulation or exceeds the permitted use, you will need to obtain permission directly from the copyright holder. To view a copy of this licence, visit http://creativecommons.org/licenses/by$\mathrm{nc} / 4.0 /$.

\section{REFERENCES}

1. World Health Organization. WHO guidelines for the treatment of Neisseria gonorrhoeae: World Health Organization; 2016.

2. Jungmann E, Ison C, Martin I, Fenton K. Gonorrhoea in young heterosexuals attending urban STD clinics in Britain: a cross-sectional survey. Int J STD AIDS. 2004;15(4):243-8.

3. Kent CK, Chaw JK, Wong W, et al. Prevalence of rectal, urethral, and pharyngeal chlamydia and gonorrhea detected in 2 clinical settings among men who have sex with men: San Francisco, California, 2003. Clin Infect Dis. 2005;41(1):67-74.

4. Morris SR, Klausner JD, Buchbinder SP, et al. Prevalence and incidence of pharyngeal gonorrhea in a longitudinal sample of men who have sex with men: the EXPLORE study. Clin Infect Dis. 2006;43(10):1284-9.

5. Centers for Disease Control and Prevention (CDC) Gonorrhea-CDC Fact Sheet 2014. https://www. cdc.gov/std/gonorrhea/stdfact-gonorrhea.htm. Accessed 23 Mar 2020.

6. Rijksinstituut voor Volksgezondheid en Milieu (RIVM) Gonorroe richtlijn [Gonorrhoea guideline] 2020 . https://lci.rivm.nl/richtlijnen/gonorroe. Accessed 10 Dec 2020.

7. Rijksinstituut voor Volksgezondheid en Milieu (RIVM) Chlamydia trachomatis en lymfogranuloma venereum richtlijn [Chlamydia trachomatis and lymphogranuloma venereum guideline] 2020 . Accessed 10 Dec 2020.

8. Centers for Disease Control and Prevention (CDC) Sexually Transmitted Disease Surveillance 2018Women and infants 2018. https://www.cdc.gov/ std/stats18/womenandinf.htm. Accessed 15 July 2020.

9. Unemo M, Seifert HS, Hook EW 3rd, Hawkes S, Ndowa F, Dillon JR. Gonorrhoea. Nat Rev Dis Primers. 2019;5(1):79.

10. Reekie J, Donovan B, Guy R, et al. Hospitalisations for pelvic inflammatory disease temporally related to a diagnosis of Chlamydia or gonorrhoea: a retrospective cohort study. PLoS ONE. 2014;9(4): e94361. 
11. Centers for Disease Control and Prevention (CDC). Antibiotic resistance threats in the United States, 2019. Atlanta: U.S. Department of Health and Human Services, CDC; 2019.

12. Chesson HW, Kirkcaldy RD, Gift TL, Owusu-Edusei $\mathrm{K} \mathrm{Jr}$, Weinstock HS. An illustration of the potential health and economic benefits of combating antibiotic-resistant gonorrhea. Sex Transm Dis. 2018;45(4):250.

13. Gottlieb SL, Jerse AE, Delany-Moretlwe S, Deal C, Giersing BK. Advancing vaccine development for gonorrhoea and the Global STI Vaccine Roadmap. Sex Health. 2019;16(5):426-32.

14. Samkange-Zeeb FN, Spallek L, Zeeb H. Awareness and knowledge of sexually transmitted diseases (STDs) among school-going adolescents in Europe: a systematic review of published literature. BMC Public Health. 2011;11(1):727.

15. Chandler J, Cumpston M, Li T, Page MJ, Welch VA. Cochrane handbook for systematic reviews of interventions. New York: Wiley; 2019.

16. Moher D, Liberati A, Tetzlaff J, Altman DG. Preferred reporting items for systematic reviews and meta-analyses: the PRISMA statement. Ann Intern Med. 2009;151(4):264-9.

17. Centers for Disease Control and Prevention (CDC) Chlamydia-CDC Fact Sheet 2014. https://www. cdc.gov/std/chlamydia/stdfact-chlamydia.htm. Accessed 23 Mar 2020.

18. Rijksinstituut voor Volksgezondheid en Milieu (RIVM) Richtlijn Chlamydia trachomatis en lymfogranuloma venereum [Guideline Chlamydia trachomatis and lymphogranuloma venereum] 2020. https://lci.rivm.nl/richtlijnen/chlamydia-

trachomatis-en-lymfogranuloma-venereum. Accessed 10 Dec 2020.

19. Ekwo EE, Gosselink CA, Woolson R, Moawad A. Risks for premature rupture of amniotic membranes. Int J Epidemiol. 1993;22(3):495-503.

20. Heumann CL, Quilter LA, Eastment MC, Heffron R, Hawes SE. Adverse birth outcomes and maternal Neisseria gonorrhoeae infection: a population-based cohort study in Washington State. Sex Transm Dis. 2017;44(5):266-71.

21. Amstey MS, Steadman KT. Asymptomatic gonorrhea and pregnancy. J Am Vener Dis Assoc. 1976;3(1):14-6.

22. Hill MG, Menon S, Smith S, Zhang H, Tong X, Browne PC. Screening for Chlamydia and gonorrhea cervicitis and implications for pregnancy outcome. Are we testing and treating at the right time? J Reprod Med. 2015;60(7-8):301-8.

23. Liu B, Roberts CL, Clarke M, Jorm L, Hunt J, Ward J. Chlamydia and gonorrhoea infections and the risk of adverse obstetric outcomes: a retrospective cohort study. Sex Transm Infect. 2013;89(8):672-8.

24. Moodley D, Sartorius B, Madurai S, Chetty V, Maman S. Pregnancy outcomes in association with STDs including genital HSV-2 shedding in a South African cohort study. Sex Transm Infect. 2017;93(7):460-6.

25. Waight MT, Rahman MM, Soto P, Tran T. Sexually transmitted diseases during pregnancy in Louisiana, 2007-2009: high-risk populations and adverse newborn outcomes. J La State Med Soc. 2013;165(4):219-26.

26. Mann JR, McDermott S, Gill T. Sexually transmitted infection is associated with increased risk of preterm birth in South Carolina women insured by Medicaid. J Matern Fetal Neonatal Med. 2010;23(6): 563-8.

27. Reekie J, Roberts C, Preen D, et al. Chlamydia trachomatis and the risk of spontaneous preterm birth, babies who are born small for gestational age, and stillbirth: a population-based cohort study. Lancet Infect Dis. 2018;18(4):452-60.

28. Bleich AT, Sheffield JS, Wendel GD Jr, Sigman A, Cunningham FG. Disseminated gonococcal infection in women. Obstet Gynecol. 2012;119(3): 597-602.

29. Warr AJ, Pintye J, Kinuthia J, et al. Sexually transmitted infections during pregnancy and subsequent risk of stillbirth and infant mortality in Kenya: a prospective study. Sex Transm Infect. 2019;95(1): 60-6.

30. Stoll BJ, Kanto WP Jr, Glass RI, Pushkin J. Treated maternal gonorrhea without adverse effect on outcome of pregnancy. South Med J. 1982;75(10): 1236-8.

31. Schonfeld A, Feldt T, Tufa TB, et al. Prevalence and impact of sexually transmitted infections in pregnant women in central Ethiopia. Int J STD AIDS. 2018;29(3):251-8.

32. Germain M, Krohn MA, Hillier SL, Eschenbach DA. Genital flora in pregnancy and its association with intrauterine growth retardation. J Clin Microbiol. 1994;32(9):2162-8.

33. Laga M, Plummer FA, Nzanze H, et al. Epidemiology of ophthalmia neonatorum in Kenya. Lancet. 1986;2(8516):1145-9. 
34. Hammerschlag MR, Cummings C, Roblin PM, Williams TH, Delke I. Efficacy of neonatal ocular prophylaxis for the prevention of chlamydial and gonococcal conjunctivitis. $\mathrm{N}$ Engl J Med. 1989;320(12):769-72.

35. Mahon BE, Temkit M, Wang J, Rosenman MB, Katz BP. Pelvic inflammatory disease during the postpartum year. Infect Dis Obstet Gynecol. 2005;13(4): 191-6.

36. Plummer FA, Laga M, Brunham RC, et al. Postpartum upper genital tract infections in Nairobi, Kenya: epidemiology, etiology, and risk factors. J Infect Dis. 1987;156(1):92-8.

37. Sorensen HJ, Mortensen EL, Reinisch JM, Mednick SA. Association between prenatal exposure to bacterial infection and risk of schizophrenia. Schizophr Bull. 2009;35(3):631-7.

38. Ness RB, Smith KJ, Chang CC, Schisterman EF, Bass DC. Prediction of pelvic inflammatory disease among young, single, sexually active women. Sex Transm Dis. 2006;33(3):137-42.

39. Plummer FA, Chubb H, Simonsen JN, et al. Antibodies to opacity proteins (Opa) correlate with a reduced risk of gonococcal salpingitis. J Clin Investig. 1994;93(4):1748-55.

40. Laga M, Manoka A, Kivuvu M, et al. Non-ulcerative sexually transmitted diseases as risk factors for HIV1 transmission in women: results from a cohort study. AIDS. 1993;7(1):95-102.

41. Wiesenfeld HC, Hillier SL, Meyn LA, Amortegui AJ, Sweet RL. Subclinical pelvic inflammatory disease and infertility. Obstet Gynecol. 2012;120(1):37-43.

42. Campbell S, Lynch J, Esterman A, McDermott R. Pre-pregnancy predictors linked to miscarriage among Aboriginal and Torres Strait Islander women in North Queensland. Aust N Z J Public Health. 2011;35(4):343-51.

43. Rich E, Hook EW 3rd, Alarcon GS, Moreland LW. Reactive arthritis in patients attending an urban sexually transmitted diseases clinic. Arthritis Rheum. 1996;39(7):1172-7.

44. Reekie J, Donovan B, Guy R, et al. Risk of pelvic inflammatory disease in relation to chlamydia and gonorrhea testing, repeat testing, and positivity: a population-based cohort study. Clin Infect Dis. 2018;66(3):437-43.

45. Lee NC, Rubin GL, Grimes DA. Measures of sexual behavior and the risk of pelvic inflammatory disease. Obstet Gynecol. 1991;77(3):425-30.
46. Reekie J, Donovan B, Guy R, Hocking JS, Kaldor JM, Mak D, Preen D, Ward J, Liu B. Chlamydia and reproductive health outcome investigators. Risk of ectopic pregnancy and tubal infertility following gonorrhea and chlamydia infections. Clin Infect Dis. 2019;69(9):1621-3. https://doi.org/10.1093/ $\mathrm{cid} / \mathrm{ciz} 145$.

47. Sherman KJ, Daling JR, Stergachis A, et al. Sexually transmitted diseases and tubal pregnancy. Sex Transm Dis. 1990;17(3):115-21.

48. Sherman KJ, Daling JR, Weiss NS. Sexually transmitted diseases and tubal infertility. Sex Transm Dis. $1987 ; 14(1): 12-6$.

49. Grodstein F, Goldman MB, Cramer DW. Relation of tubal infertility to history of sexually transmitted diseases. Am J Epidemiol. 1993;137(5):577-84.

50. Cates W Jr, Joesoef MR, Goldman MB. Atypical pelvic inflammatory disease: can we identify clinical predictors? Am J Obstet Gynecol. 1993;169(2 Pt 1):341-6.

51. Newman DR, Rahman MM, Brantley A, Peterman TA. Rates of new Human Immunodeficiency Virus (HIV) diagnoses after reported sexually transmitted infection in women in Louisiana, 2000-2015: implications for HIV prevention. Clin Infect Dis. 2020;70(6):1115-20. https://doi.org/10.1093/cid/ ciz303.

52. Peterman TA, Newman DR, Maddox L, Schmitt K, Shiver S. Risk for HIV following a diagnosis of syphilis, gonorrhoea or chlamydia: 328,456 women in Florida, 2000-2011. Int J STD AIDS. 2015;26(2): 113-9.

53. Wang YC, Chung $\mathrm{CH}$, Chen $\mathrm{JH}$, et al. Gonorrhea infection increases the risk of prostate cancer in Asian population: a nationwide population-based cohort study. Eur J Clin Microbiol Infect Dis. 2017;36(5):813-21.

54. Sarma AV, McLaughlin JC, Wallner LP, et al. Sexual behavior, sexually transmitted diseases and prostatitis: the risk of prostate cancer in black men. J Urol. 2006;176(3):1108-13.

55. Sutcliffe S, Giovannucci E, De Marzo AM, Leitzmann MF, Willett WC, Platz EA. Gonorrhea, syphilis, clinical prostatitis, and the risk of prostate cancer. Cancer Epidemiol Biomark Prev. 2006;15(11):2160-6.

56. Sutcliffe S, Giovannucci E, De Marzo AM, Willett WC, Platz EA. Sexually transmitted infections, prostatitis, ejaculation frequency, and the odds of lower urinary tract symptoms. Am J Epidemiol. 2005;162(9):898-906. 
57. Barbee LA, Khosropour CM, Dombrowksi JC, Golden MR. New human immunodeficiency virus diagnosis independently associated with rectal gonorrhea and chlamydia in men who have sex with men. Sex Transm Dis. 2017;44(7):385-9.

58. Beymer MR, Weiss RE, Halkitis PN, et al. Disparities within the disparity-determining HIV risk factors among Latino gay and bisexual men attending a community-based clinic in Los Angeles, CA. J Acquir Immune Defic Syndr. 2016;73(2):237-44.

59. Craib KJ, Meddings DR, Strathdee SA, et al. Rectal gonorrhoea as an independent risk factor for HIV infection in a cohort of homosexual men. Genitourin Med. 1995;71(3):150-4.

60. Pathela P, Braunstein SL, Blank S, Schillinger JA. HIV incidence among men with and those without sexually transmitted rectal infections: estimates from matching against an HIV case registry. Clin Infect Dis. 2013;57(8):1203-9.

61. Katz DA, Dombrowski JC, Bell TR, Kerani RP, Golden MR. HIV incidence among men who have sex with men after diagnosis with sexually transmitted infections. Sex Transm Dis. 2016;43(4): 249-54.
62. Tilchin C, Schumacher CM, Psoter KJ, et al. Human immunodeficiency virus diagnosis after a syphilis, gonorrhea, or repeat diagnosis among males including non-men who have sex with men: what is the incidence? Sex Transm Dis. 2019;46(4):271-7.

63. Beck EJ, Mandalia S, Leonard K, Griffith RJ, Harris JR, Miller DL. Case-control study of sexually transmitted diseases as cofactors for HIV-1 transmission. Int J STD AIDS. 1996;7(1):34-8.

64. Gottlieb SL, Ndowa F, Hook EW 3rd, et al. Gonococcal vaccines: public health value and preferred product characteristics; report of a WHO global stakeholder consultation, January 2019. Vaccine. 2020;38(28):4362-73.

65. Lian W-Q, Luo F, Song X-L, Lu Y-J, Zhao S-C. Gonorrhea and prostate cancer incidence: an updated meta-analysis of 21 epidemiologic studies. Med Sci Monit Int Med J Exp Clin Res. 2015;21: 1902-10.

\section{Publisher's Note}

Springer Nature remains neutral with regard to jurisdictional claims in published maps and institutional affiliations. 